\title{
Design and Simulation studies of an Ionization Cooling channel using lithium lenses and solenoid transport channels.*
}

\author{
$\underline{\text { P. Spentzouris }}^{\dagger}$, D. Neuffer, FNAL, Batavia, IL
}

\begin{abstract}
The successful design of a high luminosity muon collider requires that the six dimensional phase-space of the muon beam generated by pion decay be reduced by a factor of $\sim\left(10^{5}-10^{6}\right)$ before it enters the collider. This "beam cooling" has to take effect in a time scale comparable to the lifetime of the muon. Because of this time limitation, Ionization Cooling is the preferred option for the muon collider. In this paper we discuss the design issues for an Ionization Cooling channel based on lithium lenses and solenoid transport channels and we present simulation results obtained using a detailed tracking code with a complete model of muon-atom interactions. Cooling results for a minimal configuration of two lithium lenses and one matching section are presented, for beams with a normalized transverse emittance of $0.0067 \mathrm{~m}$-rad. The cooling factor obtained is $\sim 1.5$.
\end{abstract}

\section{INTRODUCTION}

In the current muon collider scenarios [1] the 6-D phasespace reduction required to obtain a high luminosity collider is on the order of $10^{5}-10^{6}$. This "beam cooling" must be obtained in time scales on the order of the muon lifetime ( $\sim 2 \mu \mathrm{s})$. The technique which in principle could accomplish this task, within the required time window, is ionization cooling [2,3]. In ionization cooling the beam, while passing through material, loses both transverse and longitudinal momentum by ionization loss $(\mathrm{dE} / \mathrm{dx})$. The longitudinal momentum is then restored by passing the beam through acceleration cavities. Since Multiple Coulomb Scattering in the material constitutes a heating term, the absorbers have to be placed in strong focusing field to minimize this contribution. Note that this method in its simple form cools only in the transverse dimensions of the 6-D phase space. A complete discussion for 6-D designs is presented in reference [1].

Cooling by large factors requires many successive stages of energy loss and acceleration. In this paper we discuss specific implementations of ionization cooling using lithium lenses as the cooling elements, and solenoids as the transport and matching elements. The performance of such channels depends critically on the accurate modeling of ionization and multiple scattering, and the beam emittance involved is so large that the par-axial approximation is inadequate. In order to obtain realistic modeling, we base the simulations of our design on the GEANT3 package [4], a detailed tracking code, with a complete implementation of

\footnotetext{
* Work supported by the U.S. Department of Energy.

† Email: spentz@fnal.gov
}

muon interactions in matter. In addition, the field maps required correspond to the actual geometry of the elements of the beamline, including fringe fields. Finally, in order to maintain high accuracy in computing traces over large distances, the precision of this package has been upgraded to double precision, DPGeant package [5].

\section{DESIGN PARAMETERS AND CONSTRAINS}

The use of an active lens absorber (such as a Li lens) in ionization cooling seems to be very advantageous. The azimuthal magnetic field, $B_{\theta}=\frac{\mu I r}{2 \pi R^{2}}$ in a cylindrical conductor of radius $\mathrm{R}$ provides linear radial focusing, with an equilibrium betatron function of $\beta=\left(B \rho / B^{\prime}\right)^{1 / 2}$, where $B^{\prime}$ is the field gradient. For a nominal beam momentum of 300 $M e V / c$ and $B^{\prime}=1000 T / m, \beta$ of $\sim 0.03 m$ is obtained. This $\beta$, together with the radiation length and $d E / d x$ values for lithium makes Li lenses very efficient ionization cooling devices (see reference [1] for a discussion on various ionization cooling options). The main issue for constructing a cooling channel based on Li lenses is optics, since following each active lens the muons have to be accelerated, and the matching section between the lens and the accelerating section has to be short, in order to avoid decay losses. In addition, energy straggling in the absorber dilutes the longitudinal phase space. In our design we use 1/4 Larmor wavelength $\left(\lambda_{L}\right)$ long solenoid lenses to match in and out of the accelerating section, which consists of $805 \mathrm{M} \mathrm{Hz}$ open cell RF cavities embedded in a weak solenoidal field. The $\lambda_{L} / 4$ length requirement is imposed to provide minimum beam divergence into the acceleration channel. For particles with the design momentum the transverse focusing is essentially point to parallel, $\alpha=0$, entering the acceleration channel, and parallel to point exiting it (entering the next Li lens). No chromatic fitting has been performed at this stage of the simulation $\left(\frac{\partial \beta}{\partial \frac{\delta p}{p}}=0, \frac{\partial \alpha}{\partial \frac{\delta p}{p}}=0\right)$. The length of the solenoid in the acceleration section is constrained to be an integral number of Larmor wavelengths. The apertures and the field strengths are selected to match (or at least accept) the incoming beam parameters, so they vary accordingly depending on the particular configuration. The maximum field strengths used are limited to values that could be obtained either with currently available technology or with the completion of ongoing R\&D efforts [6]. The RF frequency is constrained by the high gradient requirements $(30 \mathrm{MV} / \mathrm{m})$ of the channel. In principle, lower frequency RF provides more flexibility in the design of such channels, but high gradients are not currently feasible at low RF frequencies. In order to control the longitudinal phase space growth, the choice of RF frequency constrains the beam en- 
ergy to be higher than the optimal for transverse cooling [7].

\section{SIMULATION RESULTS}

The first implementation of our design was for a beam with a transverse normalized emittance of $6.7 \mathrm{~mm}$-rad. This example corresponds to an "upstream" cooling stage of the baseline muon collider design [1]. The design parameters of this channel were first obtained using a matrix representation of the focusing elements [8]. These values were then used to generate the realistic fields and geometric description used in the detailed DPGeant simulation. They were optimized by using the optimization package Minuit [9] which iterated the passage of 500 muons selected to cover the beam phase space through the fully simulated channel. The channel parameters are tabulated in table 1 . The section simulated consisted of 2 lithium lenses ( 1 and 2 in the table), a matching section with 2 strong solenoid lenses (front and back), and a solenoid transport section where the RF cavities were placed. These RF cavities were divided into 3 groups, the first and last used for longitudinal focusing, and the second for acceleration. The RF characteristics are shown in table 2 and the accelerating field is $30 \mathrm{MV} / \mathrm{m}$. The beam injected into this channel has momentum of 473 $\mathrm{MeV} / \mathrm{c}$, with transverse momentum r.m.s size of $32 \mathrm{MeV} / \mathrm{c}$, beam radius r.m.s of $2.2 \mathrm{~cm}$, r.m.s bunch length of $1.5 \mathrm{~cm}$ and a r.m.s energy spread of $16 \mathrm{MeV}$. The results of the sim-

Table 1: Channel Specifications

\begin{tabular}{|l|c|c|c|}
\hline & Field (T) & Length $(\mathbf{m})$ & Diameter $(\mathrm{m})$ \\
\hline LiLens1 & 1.3 & 1.8 & 0.176 \\
Sol Front & 2.5 & 1.24 & 0.39 \\
Sol Transport & 0.78 & 10 & 0.51 \\
Sol Back & 3.3 & 1.45 & 0.39 \\
LiLens 2 & 1.62 & 1.8 & 0.142 \\
\hline
\end{tabular}

ulation are shown in figures 1 - 4. In figure 1 the normalized transverse emittance and transmission of the channel are plotted as a function of position along the beamline. The emittance is reduced in the first $\mathrm{Li}$ lens from $0.0067 \mathrm{~m}-\mathrm{rad}$ to $0.0052 \mathrm{~m}$-rad, stays constant in the matching section and acceleration channel, and then is reduced again in the second lens to $0.0047 \mathrm{~m}$-rad. The losses are only non-decay (muon decays were turned off during this simulation) and they are about $1 \%$ inside the first Li lens (due to large angle scatters from particles on the tail of the beam distributions), and on the order of $5 \%$ in the second Li lens because of the development of additional tails in the matching and accelerating sections. The beam envelope is shown in figure 2, together with the average $\beta \gamma$ of the beam. The second order correlation coefficients are shown in figure 3 . As expected, the $\left(P_{x}, y\right)$ and $\left(P_{y}, x\right)$ correlations are maximal and opposite in sign inside the solenoid, while the $\left(P_{x}, x\right)$ and $\left(P_{y}, y\right)$ are equal and show the transverse motion properties of the
Table 2: RF Specifications

\begin{tabular}{|l|c|c|}
\hline & Length (m) & Stable Phase (degrees) \\
\hline Focus1 & 2.84 & 6 \\
Acceleration & 4.8 & 90 \\
Focus2 & 2.4 & 4.4 \\
\hline
\end{tabular}

beam in the matching section. All other correlations are zero, except the $\left(P_{z}, c t\right)$ correlation which shows the effects of the focusing and acceleration RF sections on the beam. For the all of the above plots, the points on the horizon-
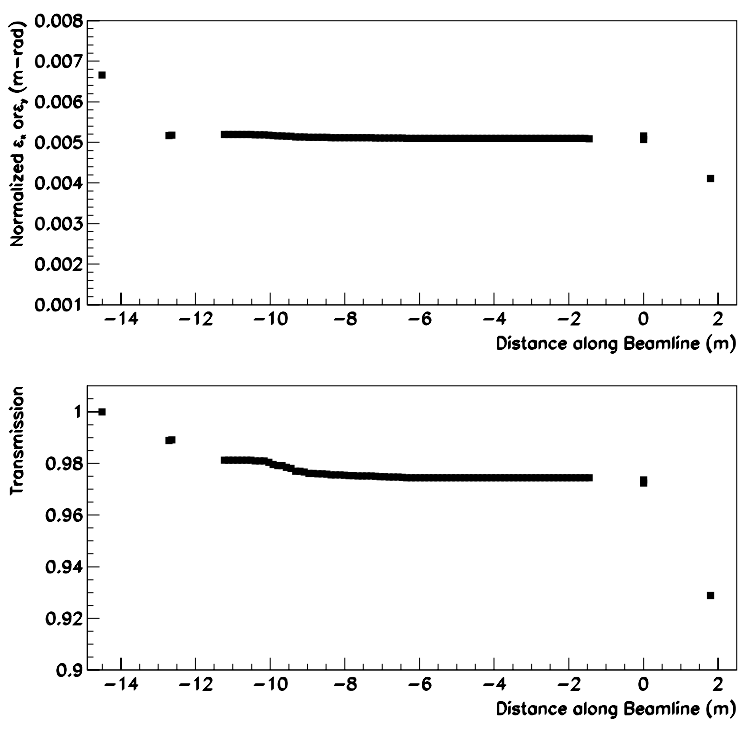

Figure 1: Transverse normalized emittance (top) and transmission (bottom) as a function of the longitudinal position along the channel.

tal axis correspond to the entrance and exit of the first $\mathrm{Li}$ lens, the entrance and exit of the first matching solenoid, the middle of each RF cell, the entrance and exit of the second matching solenoid, and the entrance and exit of the second lithium lens. The beam phase space is described using the transverse variables $x, P_{x}, y, P_{y}$, and the longitudinal variables $c t$ and $P_{z}$. The emittance is calculated according to the statistical (rms) definition [10]. Since in the presence of an axial magnetic field there are correlations between $\left(x, P_{y}\right)$ and $\left(y, P_{x}\right)$ in the lab frame, the normalized transverse emittance is calculated using the determinant of the $4 \times 4$ covariance matrix of the transverse coordinates. The values shown in figure 1 are calculated from the square root of the above determinant: $\epsilon_{x} \times \epsilon_{y}=(\text { det })^{1 / 2} / m_{0}^{2}$. Since the beam is symmetric, the correlations mentioned above exactly cancel (figure 3 ), and $\epsilon_{x}=\epsilon_{y}$. The longitudinal phase space of the beam at the entrance of the first Li lens and at the exit of the second Li lens is shown in figure 4. There is some tail development, but overall the longitudi- 

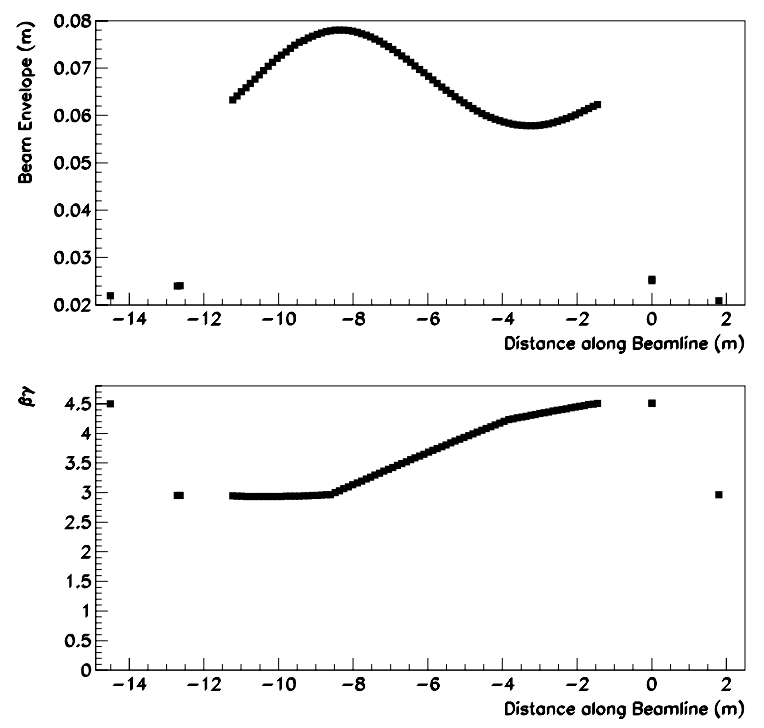

Figure 2: Beam envelope (top) and $\beta \gamma$ (bottom) as a function of the longitudinal position along the channel.

nal phase space is kept under control. The same calcula-
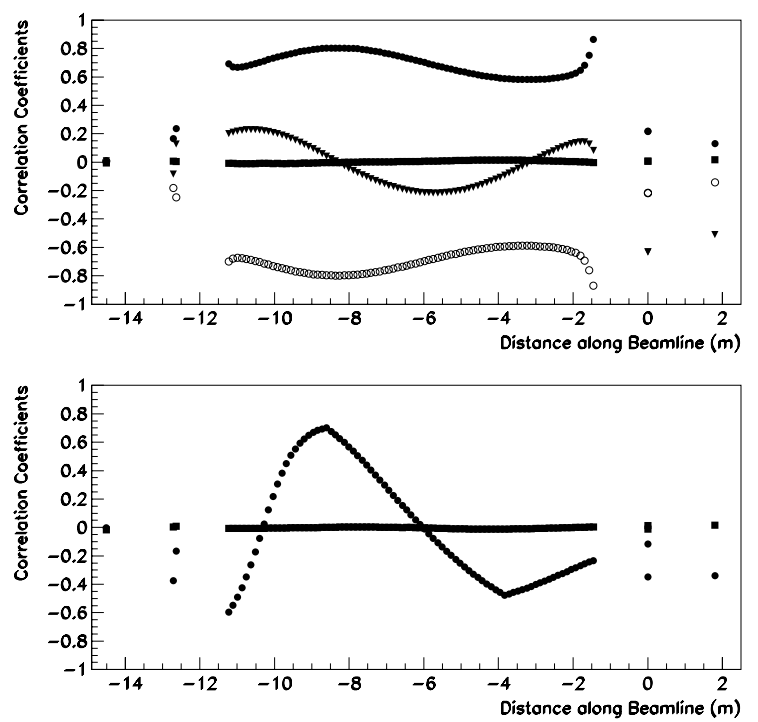

Figure 3: Correlation coefficients for $P_{x}$ and $P_{y}$ (top) and $P_{z}$ (bottom) as a function of the longitudinal position along the channel. The only non zero correlations are for $P_{x} \mathrm{y}$ (bullets), $P_{y}$ x (open symbol), $P_{x} \mathrm{x}$ and $P_{y}$ y (filled triangles), and $P_{z}$ ct (bullets).

tion was repeated for a smaller beam (normalized transverse emittance $\sim 0.00061 \mathrm{~m}$-rad), after scaling the strengths and radii of the lenses and the phase of the focusing and accelerating sections. The cooling results were similar, but the losses were much larger $(\sim 25 \%)$, mostly due to amplified chromatic effects.

In summary, we have designed and simulated in full detail an ionization cooling channel which consists of two lithium lenses a solenoidal matching section, and an RF section at $805 \mathrm{MHz}$. The transverse cooling factor obtained for $473 \mathrm{MeV} / \mathrm{c}$ muons with a normalized transverse emittance of $0.0067 \mathrm{~m}$-rad is $\sim 1.5$, while the longitudinal emittance remains under control, and the losses are at the $7 \%$ level.
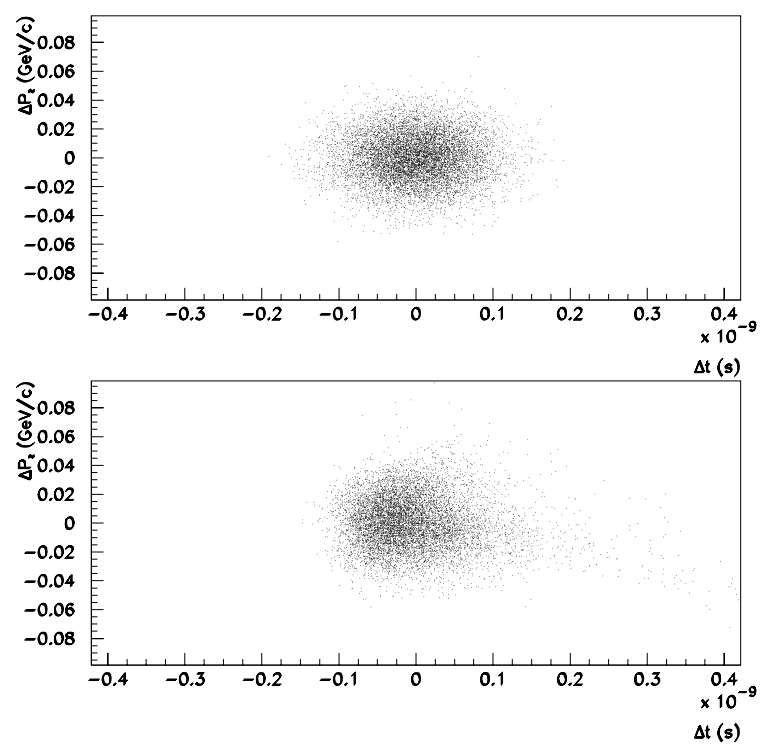

Figure 4: The Beam longitudinal phase space $\left(P_{z}\right.$ vs t) at the entrance of the channel (top) and at the exit (bottom).

\section{REFERENCES}

[1] Status of the muon Collider Research and Development and Future Plans BNL-65-623, Fermilab -PUB-98/179, LBNL41935, submitted to Phys. Rev. ST Accel. Beams.

[2] G.I.Budker and A.N.Skrinsky, Electron cooling and new possibilities in elementary particle physics, SoV. Phys. Usp. 21 277 (1978).

[3] D.Neuffer, Particle Accelerators 1475 (1983).

[4] Geant Manual V3.2.2.1, CERN Program Library W5013.

[5] See P.Lebrun's contribution in these proceedings.

[6] G.Silvestrov, in Proceedings of the 9th Advanced ICFA Beam Dynamics Workshop, editor J.C.Gallardo (AIP Press, Woodbury, New York, 1996), Vol. 372.

[7] B.Palmer and R.Fernow, Cooling notes, USPAS, January 1999, http://pubweb.bnl.gov/people/palmer/course/

[8] V. Balbekov, $805 \mathrm{MHz}, 30 \mathrm{MV} / \mathrm{m}$, Li-lenses based cooling channel, internal Muon Collider report, unpublished.

[9] Minuit Reference Manual, V94.1, CERN Program Library Writeup D506.

[10] P.M.Lapostolle, IEEE Trans. Nucl. Sci NS-18, No 3, 1101 (1971) 